\title{
Supervision of loT Water Pump Remotely through Versatile Media
}

\author{
A. S. Zaforullah Momtaz \\ Assistant Professor, Computer \\ Science \& Engineering \\ University of Asia Pacific (UAP)
}

\author{
Nadeem Ahmed \\ Assistant Professor, Computer \\ Science \& Engineering \\ University of Asia Pacific (UAP)
}

\author{
Rahul Acharjee \\ Student, Computer Science \& \\ Engineering \\ University of Asia Pacific (UAP)
}

\begin{abstract}
This paper presents a technique to operate different kind of electrical machine in an automated way through Bluetooth or Internet. That obvious means user can operate their required machine from long distance. To the user, this system will provide as a $\mathrm{P} 2 \mathrm{P}$ device controller, where the user can plug any types of machine and can trigger via internet based communication medium. Also the controller provides the user same facilities in Bluetooth medium when he is available in a short distance. Again in the proposed system, we are controlling over IP, so in a single time multi-user control issue also can be avoided. In practical state, our target machine was only water pump as it may need to control from any kind of distance besides controlling it using AC/DC or virtual switches as well as voice command based on smart phone applications.
\end{abstract}

\section{Keywords}

Arduino, Bluetooth, CDMA, GSM, IP, P2P, Wi-Fi, Ethernet, GSM, IoT

\section{General Terms}

Arduino UNO R3, Button Shield, Bluetooth Shield, Relay Shield, Submersible, pump, automation

\section{INTRODUCTION}

An automated system is a kind of system where the input is commanded and the system carries out a process to generate production [1] as required. The idea of "automation" has been existed for a number of eras. It commenced with a student connecting two electric wires to the hands of an alarm clock in order to close a circuit of a battery and light bulb. But later, different companies have developed automated systems of their own [2] for various purposes. The automated system are providing advantages like, process information quickly than human beings, can do the assigned task repeatedly without getting bored, needing breaks or making mistakes, can do very detailed work and follow the given instructions without error, can work in places where it would be unsafe to put a human, get more done than humans but cost less to operate, and can be reprogrammed to do various tasks [3].

Now a day, IoT-based solution has become a very popular technique to get control over a system through internet media. The evolution of IoT-based solutions helps to implement of access control systems in a far more standard way which also capable of resolving various limitations of existing traditional system, also carrying additional functionalities. That's why in our research work we investigate this technique to implement an automated system to control any type of machines specially water pump from versatile media.
IoT-based machine control system could be the fastest, broadest and simplest technique where the control interface could be any smart device like mobile phone, tab, laptop etc. In the proposed system, control is obtaining through user account to maintain the communication in between the user who wants to control it and the server; also the server and the water pump itself. The user will provide the necessary triggering instruction to the server like the trigger on or off of the machine or according to the system-specified time schedule. Once the server receives the instruction from the authorized user and it will carry the instruction to the load controller. And all these tasks will be done through the internet communication media. In practical state, we have performed experiments on a submersible water pump according to user's provided instruction. Figure 1 demonstrates the whole system structure chain among two different users, server, and three different types of IoT-based machine. According to this figure, 'User 1' is authorized to communicate with 'Machine 2' and 'Machine 3', on the other hand 'User 2' is authorized to communicate with 'Machine 1'. The duty of server is to create the bridge for all of these clients.

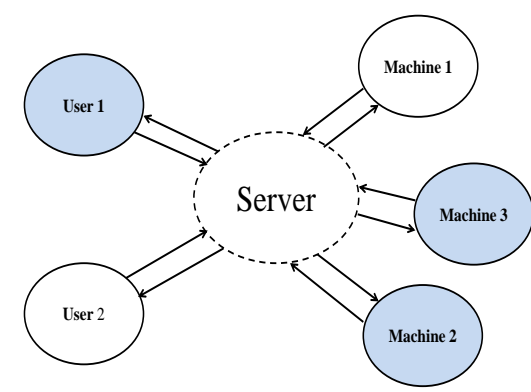

Figure 1: System Structure chain

'User 1' can directly communicate with 'Machine 2' as well as 'Machine 3' over P2P [4] means 'User 1':'Machine 2' and again 'User 1':'Machine 3'. Same communication is applicable for 'User 2':'Machine 1'. Therefore it's quite clear that without assigned authorization one user can't access the machine of others. This authorization process is maintained by the server.

In the practical state, the proposed water pump can be commanded through a web application titled 'IP Machine' [5] which is hosted in 'Quizee Club' [6], which is basically a web server that hosts the necessary database [7], provides the communication platform among users and the machines for this project. The pump can also be commanded using a smart phone as it has a Bluetooth shield [8] itself along with the GSM shield [9]. Thus the user command can be taken in different ways like pressing buttons, timer, and vocal 
command on that smart phone. For these purpose the smart phone requires an app titled 'Broxcode' [10], which is capable to contact with the Bluetooth module to perform the expected local commands. The provided signal to the Bluetooth shield is a set of characters meaning string [11] type data. Once the Arduino board gets command, it triggers the pump.

\section{RELATED LITERATURE}

The project 'Controlling Water Pump' using 'Cellular Mobile Telephony' [12], authors has implemented such system via Cellular Mobile Telephony that works based on mobile phone call. The system has itself a mobile phone. When it receives a phone call an output has taken from the mobile vibration motor. This output voltage goes directly to an ADC pin of the Microcontroller as an input signal. Providing a single phone call to the system mobile phone, the vibration motor produces four discrete signals repeatedly. This output has been considered as toggle input command to the Microcontroller. Thus microcontroller triggers the relay [13] module to switching on or off the pump. Again when giving another phone call to the circuit mobile phone then the command goes to the Relay via the microcontroller and then the Relay switch on or off the pump again.

There are several limitations exist in this project like:

a) System dependent mobile phone battery needs be charged always;

b) Mobile phone connection must be connected to the a valid network;

c) Mobile operator company network must have the coverage in that area;

d) Most importantly this system can't handle multiuser control issue in a single time.

In our proposed system the dependency on mobile phone call is totally transferred to Internet as well as Bluetooth communication. The key point of this project is the capability of dealing the multi-user access issues at the same time. That's why P2P communication through web server has been introduced in the proposed system which ensures that in a single time, only the authenticated user can only access the system.

\section{PROPOSED AUTOMATED SYSTEM}

The proposed automated water pump is not only controllable via internet as well as Bluetooth but also resist to unauthorized users access. The basic features of this system are:

a) Can be controlled through web app;

b) The web app will automatically maintain the log;

c) Can be controlled locally using a smart phone along with the conventional switch;

d) Can be controlled locally using a smart phone along with the conventional switch;

e) Local user command could be as like as following:
a. Voice input
b. Virtual button input

In practical state, this system is maintained by Arduino Uno R3 [14] board which acts as the central working unit and connects all the necessary electronic shields like Bluetooth,
GSM, Ethernet, Button, Relay and others. The core of the Arduino Uno R3 is a microcontroller 'ATMEGA328' [?], which takes it command either from the Bluetooth shield or from the Internet Shields [15] or from the Button Shield. After getting the command, it provides the necessary triggering command the Relay Shield to switch on or off the pump demonstrated in Fig-2.

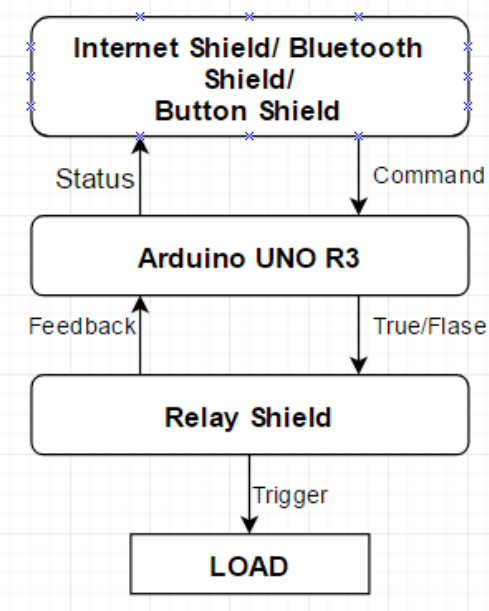

Figure 2: Command-Response Chain

After triggering the pump, the Arduino board sends the feedback to the all user media about the current status of the pump whether it is switched on or off. For a TRUE type command the pump goes into switched on state. And for a FALSE type command the pump goes into switched off state. The entire working activities inside the ATMEGA328 microcontroller is explained graphically presented in figure 3 .

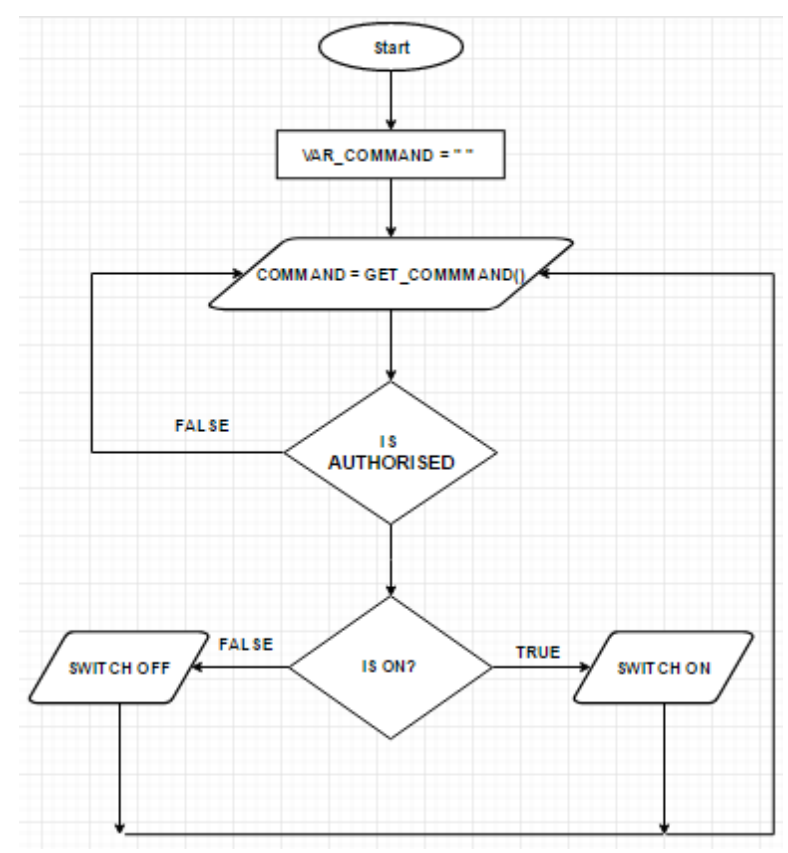

Figure 3: Automated Pump Control System

The automated pump control system that is shown on the figure 3 described the sequence of working flow of this system. First of all, the command is empty and secondly the system got the command from the shield such as Button 
Shield, Bluetooth Shield and Internet Shield. After getting the command, the system checks it is authorized or not. If it returns true then it will check that the system is on or off. The system will be on if it is off otherwise it will be off. After getting all commands, The system will go to the $2^{\text {nd }}$ step and works sequentially as well as it is enable to getting the input command from the different shield like Button shield, Bluetooth shield and Internet shield. The procedure of the work flow has been written below as a pseudo code:

$$
\begin{gathered}
\text { Var command = " " } \\
\text { Command = getCommand() } \\
\text { IF is Authorized THEN } \\
\text { IF is on THEN } \\
\text { ELSE Switch on } \\
\text { END IF }
\end{gathered}
$$$$
\text { END IF }
$$

\subsection{Button Shield Operation}

In the prototype, a button shield having 4 switches has been introduced to control the pump in a digital fashion. Out of these 4 switches only 1 has been used.

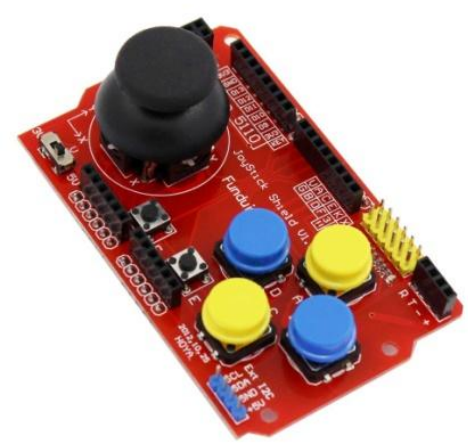

Figure 4: Button Shield

The working activity of the button is pretty simple. When the target button is pressed it sends a high signal to the Arduino Board at the digital pin\#3. When the button is free or released it sends a low signal. It works as like as a toggle button enlisted in Table-1. Thus when the board receives high signal, then it checks the current state of the pump. If it is in on state, the board triggers the pump into off state and vice versa.

Table-1: button command to trigger the pump

\begin{tabular}{|c|c|c|}
\hline Command No & Button State & Signal Generated \\
\hline 1 & Free/Release & Low/0 \\
\hline 2 & Pressed & High/1 \\
\hline
\end{tabular}

\subsection{Bluetooth Shield Operation}

In the proposed system the water pump can be controlled via smart phone where an android app has been used named as Broxcode. This app is featured by Matrix tools, Arrow Keys, Terminal, Buttons, Accelerator and Voice Control.

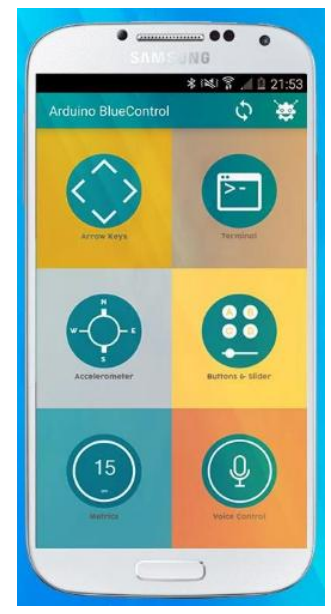

Figure 5: Broxcode App

From the Broxcode application, two features have been selected as follows:

a) Button Input: a single button command what toggles the pump from one state to another.

b) Vocal Command: two different voice input; one to switch on the pump, and the other one to switch off the pump.

There are total 6 buttons available in Broxcode named ' $A$ ', 'B', 'C', 'D', 'E', \& 'F'. Out of these 6, only the first one has been used means 'A'. And this sends a string 'toggle-pump' to the Arduino board via Bluetooth. When the board receives this string, then it checks the current state of the pump. If the pump is in on state, it triggers the pump into off state and vice versa.

Similarly the app has a built in 10 different vocal command options. Out of these 10 scopes, 4 have been used. Two of them to switch on the pump, and other two to switch off the pump shown in the following table, table-2:

Table-2: Vocal command to Trigger the Pump

\begin{tabular}{|c|c|c|}
\hline Command No & Vocal Input & For Arduino \\
\hline 1 & switch on the pump & pump-on \\
\hline 2 & switch off the pump & pump-off \\
\hline 3 & switch on the motor & pump-on \\
\hline 4 & switch off the motor & pump-off \\
\hline
\end{tabular}

\subsection{Internet Shield Operation}

The internet shield is a shield to connect an Arduino board to internet for accessing the web servers or remote work stations. In the proposed prototype different experiments have been performed with different internet shield to trigger the pump via internet. The list of internet shields is as follows:

a) Ethernet shield: it uses the RJ45 connector to connect the Arduino board with a local router which is connected to broadband internet service. Thus the Arduino board can access the workstation in wired fashion. In the experiment, 'ENC28J60' [16] has been used.

b) Wifi shield: it uses the wireless communication protocol to connect the Arduino board with a local router which is connected to broadband internet service. Thus the Arduino board can access the 
workstation in a wireless fashion. In the experiment, 'Fi250' [17] has been used.

c) GSM shield: it uses the wireless communication protocol provided by the telecom operators to connect the Arduino board to the internet. In the experiment, 'SIM900A' [18] has been used to perform this communication.

For triggering the pump from a remote place through internet media; Quizee Club has provided the necessary platform, database support, security, P2P connections as well as the web application for human interface. All this supports work in backend except the web application named 'IP Machine'. This app has two sections:

a) Switch: the switch is used to input the trigger command either in on state or in off state.

b) Machine Action: it indicates whether the pump is switched on or off in an animated fashion.

After pressing the button, the signal goes to the server and it forwards the signal to the local pump, assigned to a user particularly. After triggering the pump the Arduino board sends the server a feedback about the local action, and then the server forward this feedback to the user app. Thus the app then triggers the machine action icon as the indication of user's command.

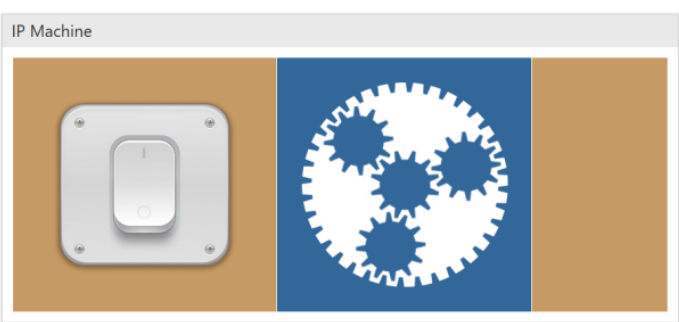

Figure 6: Web Interface for User off state

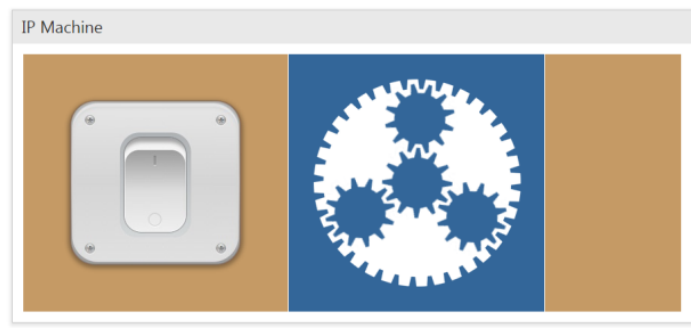

Figure 7: Web Interface for User on state

\section{HARDWARE \& SOFTWARE INTERFACE}

The user interface to control the machine from a local distance is a button shield shown in the Figure 4. In case Bluetooth, the required android app 'Broxcode' shown in the Figure 5, for controlling via internet is simply a web interface placed in Figure $7 \& 8$. All the system takes an input command from the user interface. After getting an authorized command it will trigger pump from the current state to opposite state; meaning if the pump is in off state, it will push the pump into on state and if the pump is in on state, it will push the pump into off state. After the execution of the trigger command it will send the necessary feedback to the user, as a confirmation about the execution of user's command.
The main hardware interface is shown in figure 8 , which is the first testing experiment on controlling a water pump from varsatile media. In this test experiment, button shield has been used, along with the Arduino board, relay shiled and a small submercible water pump used in labrotory tasks. This experiment is completely performed by the press and release of a selected button from the Button shiled. When the button is pressed or released it generates a signal to the Arduino board, as the button shiled is manually connected so the command is considered as an authorised input by default. In case of a high signal the Arduino tiggers the realy module to switch on/off the pump. If the pump is in off state, then it switches to on and starts to lift water from the lower tank to the desired pipe line. Again pressing the button it pushes the pump into off state, thus no water supply is obtained from the lower tank to the desired pipe line.

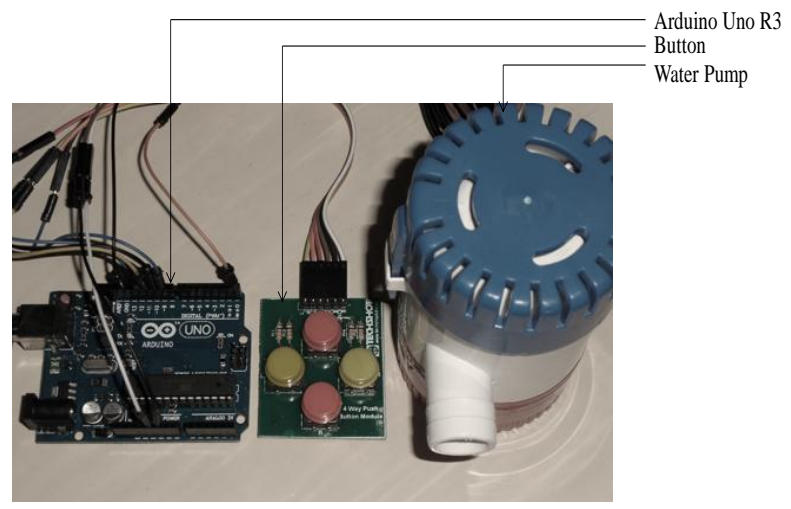

Figure 8: Hardware Interface of the automated pump

\section{LIMITATIONS \& FUTURE WORK}

Triggering a water pump from distant place is totally dependent on the internet communication channel. Internet covers a long distance; even it creates contact between two countries from far apart. But the failure of this network at any point will totally hamper the command-response procedure of the proposed project.

In case of local command via Bluetooth, distance is also another factor. The range of a standard Bluetooth communication is maximum $10 \mathrm{~m}$. For a user, out of this range is difficult to manage the pump. An automatic switching system from Bluetooth to Internet communication system can be introduced here in future to improve the facilities of this project.

A scheduling system for both of the single and multiple pumps will be very much helpful for the users. It would be a preprogrammed system, where a user will create a set of trigger time according to the necessity. Thus pump could be on or off automatically without any human intervention.

\section{ACKNOWLEDGEMENT}

"This work was supported by Software \& Hardware Club as well as Research \& Publication unit of Computer Science and Engineering Department, University of Asia Pacific, Dhaka, Bangladesh."

\section{REFERENCES}

[1] www.gordonschools.aberdeenshire.sch.uk/Departments/ Computing/StandardGrade/AutoSysWeb/1Introduction.h tm. 
[2] Armando Roy Delgado, Rich Picking and Vic Grout; Remote-Controlled Home Automation Systems with Different Network Technologies.

[3] www.gordonschools.aberdeenshire.sch.uk/Departments/ Computing/StandardGrade/AutoSysWeb/2Advantages.ht $\mathrm{ml}$.

[4] p2p link: http://www.quizee.club/url/Q.

[5] IP machine: http://www.quizee.club/url/R.

[6] Quizee Club: quizee.club.

[7] Database Server: https://www.mysql.com/.

[8] Bluetooth Shield: http://www.quizee.club/url/S.
[9] GSM Shield: http://www.quizee.club/url/T.

[10] Broxcode app: http://www.quizee.club/url/U.

[11] String Character: http://www.quizee.club/url/V.

[12] Celluler Telephony: http://www.quizee.club/url/W.

[13] Relay Module: http://www.quizee.club/url/X.

[14] Arduino UNO R3: https://www.adafruit.com/product/50.

[15] Internet Shield: http://www.quizee.club/url/Y.

[16] Ethernet Shield: http://www.quizee.club/url/Z.

[17] Wi-Fi Shield Connector: http://www.quizee.club/url/10.

[18] GSM Shield Connector: http://www.quizee.club/url/11. 